\title{
THE POTENTIALS METHOD FOR A CLOSED QUEUEING SYSTEM WITH HYSTERETIC STRATEGY OF THE SERVICE TIME CHANGE
}

\author{
Yuriy Zhernovyi ${ }^{1}$, Bohdan Kopytko ${ }^{2}$ \\ ${ }^{1}$ Ivan Franko National University of Lviv, Lviv, Ukraine \\ ${ }^{2}$ Institute of Mathematics, Czestochowa University of Technology \\ Czestochowa, Poland \\ yu_zhernovyi@yahoo.com,bohdan.kopytko@im.pcz.pl
}

\begin{abstract}
We propose a method for determining the characteristics of a single-channel closed queueing system with an exponential distribution of the time generation of service requests and arbitrary distributions of the service times. In order to increase the system capacity, two service modes (the main mode and overload mode), with the service time distribution functions $F(x)$ and $\tilde{F}(x)$ respectively, are used. The overload mode starts functioning if at the beginning of service of the next customer the number of customers in the system $\xi(t)$ satisfies the condition $\xi(t)>h_{2}$. The return to the main mode carried out at the beginning of service of the customer, for which $\xi(t)=h_{1}$, where $1 \leq h_{1}<h_{2}$. The Laplace transforms for the distribution of the number of customers in the system during the busy period and for the distribution function of the length of the busy period are found. The developed algorithm for calculating the stationary characteristics of the system is tested with the help of a simulation model constructed with the assistance of GPSS World tools.
\end{abstract}

Keywords: queueing system with a finite number of sources, hysteretic strategy for service time, potentials method, stationary characteristics

\section{Introduction}

Closed queueing systems are widely used as models to evaluate characteristics of the information systems, data networks and queueing processes in production, transport, trade, logistics and service systems [1]. The closed system is also called the system with a finite number of sources or Engset system.

Suppose that a single-channel queueing system receives service requests from $m$ identical sources. Each source is alternately on and off. A source is off when it has a service request being served, otherwise the source is on. A source in the on-state generates a new service request after an exponentially distributed time (the generation time) with mean $1 / \lambda$. The sources act independently of each other. 
The service time of a service request has an arbitrary distribution. A service request, that is generated when channel is occupied, waits in the queue.

The arrival rate of customers for a closed system at time $t$ depends on the number of customers in the system $\xi(t)$ and is equal to $\lambda(m-\xi(t))$. Such a flow of customers is called the Poisson flow arrivals of the second kind [2, p. 135].

In the case of an exponential service time distribution, the formulas are known for the stationary distribution of the number of customers for a single-channel closed system [2, p. 137], and for a multichannel closed system [3, p. 134]. In [4] using the method of embedded Markov chain, an algorithm is constructed to determine the stationary distribution of the number of customers for a single-channel closed system with an arbitrary distribution of the service time.

In order to increase the system capacity, threshold strategies of the service intensity (service time) change are used in queueing systems. In the general case, the essence of this strategy is that the service time distribution depends on the number of customers in the system at the beginning of each customer service $[5,6]$. With the help of the potentials method, we have developed an efficient algorithm for computing the stationary distribution of the number of customers in the systems with threshold functioning strategies [5-10], including a single-channel closed system with service time, dependent on the queue length [5].

The potentials method is a generalization of an approach proposed by V. Korolyuk [11] for the study of a lower semicontinuous random walk to the case of several random walks. The potential method with a single basic random walk was used to study the $\mathrm{M}^{\alpha} / \mathrm{G} / 1 / \mathrm{N}$ system with a single fixed service time distribution [12]. For the study of systems with several modes of operation, it is necessary to use as many basic random walks and their potentials as there are different modes of functioning.

Since the arrival rate of customers for a closed system depends on the number of customers in the system, using the potentials method even in the case of a conventional closed system with a single function of the service time distribution $F(x)$, for each value of the number of customers $\xi(t)=n \in\{1,2, \ldots, m\}$, it is necessary to consider a selected basic random walk.

In this paper we apply the potentials method to study a single-channel closed system with two-threshold hysteretic switching of the service times. Two service modes (the main mode and overload mode), with the service time distribution functions $F(x)$ and $\tilde{F}(x)$ respectively, are used. The overload mode starts functioning if at time $t$ of the beginning of service of the next customer, the number of customers in the system satisfies the condition $\xi(t)>h_{2}$. The return to the main mode carried out at the beginning of service of the customer, for which $\xi(t)=h_{1}$, where $1 \leq h_{1}<h_{2}<m$.

\section{Basic random walks}

Denote by $\mathbf{P}_{n}$ the conditional probability, provided that at the initial time the number of customers in the queueing system is equal to $n \in\{0,1,2, \ldots, m\}$, and 
by $\mathbf{E}(\mathbf{P})$ the conditional expectation (the conditional probability) if the system starts to work at the time of arrival of the first customer. Let $\eta(x)$ be the number of customers arriving in the system during the time interval $[0 ; x)$. Let

$$
\begin{gathered}
f(s)=\int_{0}^{\infty} e^{-s x} d F(x), \quad M=\int_{0}^{\infty} x d F(x)<\infty ; \quad \tilde{f}(s)=\int_{0}^{\infty} e^{-s x} d \tilde{F}(x), \quad \tilde{M}=\int_{0}^{\infty} x d \tilde{F}(x)<\infty, \\
\bar{F}(x)=1-F(x), \quad \overline{\tilde{F}}(x)=1-\tilde{F}(x) .
\end{gathered}
$$

For $\operatorname{Re} s \geq 0$ and $n \in\left\{1,2, \ldots, h_{2}\right\}$ consider the sequences $\pi_{n i}(s), q_{n i}(s)$ and $R_{n}(s)$, defined by the relations:

$$
\begin{aligned}
& \pi_{n i}(s)=\frac{1}{f(s)} \int_{0}^{\infty} e^{-s x} \mathbf{P}_{n}\{\eta(x)=i+1\} d F(x), \quad i \in\{-1,0,1, \ldots, m-n-1\} \\
& q_{n i}(s)=\int_{0}^{\infty} e^{-s x} \mathbf{P}_{n}\{\eta(x)=i\} \bar{F}(x) d x, \quad i \in\{0,1,2, \ldots, m-n\} ; \quad R_{n}(s)=\frac{1}{f(s) \pi_{n,-1}(s)} .
\end{aligned}
$$

Similarly, for $\operatorname{Re} s \geq 0$ and $n \in\left\{h_{1}+1, h_{1}+2, \ldots, m\right\}$ we set the sequences $\tilde{\pi}_{n i}(s)$, $\tilde{q}_{n i}(s)$ and $\tilde{R}_{n}(s)$ :

$$
\begin{aligned}
& \tilde{\pi}_{n i}(s)=\frac{1}{\tilde{f}(s)} \int_{0}^{\infty} e^{-s x} \mathbf{P}_{n}\{\eta(x)=i+1\} d \tilde{F}(x), \quad i \in\{-1,0,1, \ldots, m-n-1\} \\
& \tilde{q}_{n i}(s)=\int_{0}^{\infty} e^{-s x} \mathbf{P}_{n}\{\eta(x)=i\} \overline{\tilde{F}}(x) d x, \quad i \in\{0,1,2, \ldots, m-n\} ; \quad \tilde{R}_{n}(s)=\frac{1}{\tilde{f}(s) \tilde{\pi}_{n,-1}(s)} .
\end{aligned}
$$

The sequence $\pi_{n i}(s)\left(\tilde{\pi}_{n i}(s)\right)$ with $s>0$ and a fixed $n$ can be treated as the distribution of jumps of some lower semicontinuous random walk defined by the distribution function $F(x)(\tilde{F}(x))$ of the corresponding mode of service and probabilities $\mathbf{P}_{n}\{\eta(x)=i+1\}$.

Let $T_{i \lambda}$ denote an exponentially distributed random variable with parameter $i \lambda$. Then we have

$$
\begin{aligned}
& \mathbf{P}_{n}\{\eta(x)=0\}=\mathbf{P}\left\{T_{(m-n) \lambda}>x\right\}=e^{-(m-n) \lambda x}, \quad n \in\{1,2, \ldots, m\} ; \\
& \mathbf{P}_{n}\{\eta(x)=j\}=\mathbf{P}\left\{\sum_{i=0}^{j-1} T_{(m-n-i) \lambda}<x<\sum_{i=0}^{j} T_{(m-n-i) \lambda}\right\}= \\
& \quad=\mathbf{P}\left\{\sum_{i=0}^{j} T_{(m-n-i) \lambda}>x\right\}-\mathbf{P}\left\{\sum_{i=0}^{j-1} T_{(m-n-i) \lambda}>x\right\}=\prod_{k=0}^{j-1}(m-n-k) \times
\end{aligned}
$$




$$
\begin{gathered}
\times \sum_{i=0}^{j}(-1)^{i+j} \frac{e^{-(m-n-i) \lambda x}}{i !(j-i) !}, \quad n \in\{1,2, \ldots, m-1\}, \quad j \in\{1,2, \ldots, m-n-1\} ; \\
\mathbf{P}_{n}\{\eta(x)=m-n\}=\mathbf{P}\left\{\sum_{i=0}^{m-n-1} T_{(m-n-i) \lambda}<x\right\}=1+\sum_{i=1}^{m-n}(-1)^{i} C_{m-n}^{i} e^{-i \lambda x}, \\
n \in\{1,2, \ldots, m-1\} .
\end{gathered}
$$

With regard to (3) the expressions (1) for $\pi_{n i}(s)$ and $q_{n i}(s)$ are reduced to the form

$$
\begin{aligned}
& \pi_{n,-1}(s)=\frac{f((m-n) \lambda+s)}{f(s)} ; \\
& \pi_{n, j-1}(s)=\frac{1}{f(s)} \prod_{k=0}^{j-1}(m-n-k) \sum_{i=0}^{j}(-1)^{i+j} \frac{f((m-n-i) \lambda+s)}{i !(j-i) !}, j \in\{1,2, \ldots, m-n-1\} ; \\
& \pi_{n, m-n-1}(s)=1+\frac{1}{f(s)} \sum_{i=1}^{m-n}(-1)^{i} C_{m-n}^{i} f(i \lambda+s) ; \quad q_{n 0}(s)=\frac{1-f((m-n) \lambda+s)}{(m-n) \lambda+s} ; \\
& q_{n j}(s)=\prod_{k=0}^{j-1}(m-n-k) \sum_{i=0}^{j}(-1)^{i+j} \frac{1-f((m-n-i) \lambda+s)}{i !(j-i) !((m-n-i) \lambda+s)} \\
& q_{n, m-n}(s)=\frac{1-f(s)}{s}+\sum_{i=1}^{m-n}(-1)^{i} C_{m-n}^{i} \frac{1-f(i \lambda+s)}{i \lambda+s}
\end{aligned}
$$

where $n \in\left\{1,2, \ldots, h_{2}\right\}$. Similarly, from (2) using (3) we obtain:

$$
\begin{aligned}
& \tilde{\pi}_{n,-1}(s)=\frac{\tilde{f}((m-n) \lambda+s)}{\tilde{f}(s)}, \quad n \in\left\{h_{1}+1, h_{1}+2, \ldots, m\right\} ; \\
& \tilde{\pi}_{n, j-1}(s)=\frac{1}{\tilde{f}(s)} \prod_{k=0}^{j-1}(m-n-k) \sum_{i=0}^{j}(-1)^{i+j} \frac{\tilde{f}((m-n-i) \lambda+s)}{i !(j-i) !}, \\
& n \in\left\{h_{1}+1, h_{1}+2, \ldots, m-1\right\}, \quad j \in\{1,2, \ldots, m-n-1\} ; \\
& \tilde{\pi}_{n, m-n-1}(s)=1+\frac{1}{\tilde{f}(s)} \sum_{i=1}^{m-n}(-1)^{i} C_{m-n}^{i} \tilde{f}(i \lambda+s), \quad n \in\{1,2, \ldots, m-1\} ; \\
& \tilde{q}_{n 0}(s)=\frac{1-\tilde{f}((m-n) \lambda+s)}{(m-n) \lambda+s}, \quad n \in\left\{h_{1}+1, h_{1}+2, \ldots, m\right\} ; \\
& \tilde{q}_{n j}(s)=\prod_{k=0}^{j-1}(m-n-k) \sum_{i=0}^{j}(-1)^{i+j} \frac{1-\tilde{f}((m-n-i) \lambda+s)}{i !(j-i) !((m-n-i) \lambda+s)}, \\
& \quad n \in\left\{h_{1}+1, h_{1}+2, \ldots, m-1\right\}, \quad j \in\{1,2, \ldots, m-n-1\} ; \\
& \tilde{q}_{n, m-n}(s)=\frac{1-\tilde{f}(s)}{s}+\sum_{i=1}^{m-n}(-1)^{i} C_{m-n}^{i} \frac{1-\tilde{f}(i \lambda+s)}{i \lambda+s}, \quad n \in\left\{h_{1}+1, h_{1}+2, \ldots, m-1\right\} .
\end{aligned}
$$


Note that

$$
\lim _{s \rightarrow+0} f(s)=\lim _{s \rightarrow+0} \tilde{f}(s)=1, \quad \lim _{s \rightarrow+0} \frac{1}{1-f(s)}=M, \quad \lim _{s \rightarrow+0} \frac{1}{1-\tilde{f}(s)}=\tilde{M} .
$$

We introduce the notation:

$$
\begin{array}{lll}
\pi_{n i}=\lim _{s \rightarrow+0} \pi_{n i}(s), & q_{n i}=\lim _{s \rightarrow+0} q_{n i}(s), & R_{n}=\lim _{s \rightarrow+0} R_{n}(s), \\
\tilde{\pi}_{n i}=\lim _{s \rightarrow+0} \tilde{\pi}_{n i}(s), & \tilde{q}_{n i}=\lim _{s \rightarrow+0} \tilde{q}_{n i}(s), & \tilde{R}_{n}=\lim _{s \rightarrow+0} \tilde{R}_{n}(s) .
\end{array}
$$

With the help of equalities (1)-(6) we can obtain expressions for the members of the sequences $\pi_{n i}, q_{n i}, R_{n}, \tilde{\pi}_{n i}, \tilde{q}_{n i}$ and $\tilde{R}_{n}$. Note that $\tilde{\pi}_{m,-1}=1, \tilde{q}_{m 0}=\tilde{M}$, $\tilde{R}_{m}=1$.

\section{Distribution of the number of customers in the system during the busy period}

Denote by $\mathbf{P}_{F, n}\left(\mathbf{P}_{F, n}\right)$ the conditional probability, provided that at the initial time the number of customers of the system is equal to $n$ and the service begins with the service time distributed according to the law $F(x)(\tilde{F}(x))$.

Let $\tau(m)=\inf \{t \geq 0: \xi(t)=0\}$ denote the length of the first busy period for the considered queueing system, and for $k \in\{1,2, \ldots, m\}$

$$
\begin{aligned}
& \psi_{n}(t, k)=\mathbf{P}_{F, n}\{\xi(t)=k, \tau(m)>t\}, \quad 1 \leq n \leq h_{2} ; \\
& \tilde{\psi}_{n}(t, k)=\mathbf{P}_{F, n}\{\xi(t)=k, \tau(m)>t\}, \quad h_{1}+1 \leq n \leq m ; \\
& \varphi_{n}(t, k)= \begin{cases}\psi_{n}(t, k), \quad 1 \leq n \leq h_{2} ; \\
\tilde{\psi}_{n}(t, k), \quad h_{2}+1 \leq n \leq m ;\end{cases} \\
& \Phi_{n}(s, k)=\int_{0}^{\infty} e^{-s t} \varphi_{n}(t, k) d t, \quad \tilde{\Phi}_{n}(s, k)=\int_{0}^{\infty} e^{-s t} \tilde{\psi}_{n}(t, k) d t, \quad \operatorname{Re} s>0 .
\end{aligned}
$$

It is evident that $\varphi_{0}(t, k)=0, \tilde{\psi}_{h_{1}}(t, k)=\varphi_{h_{1}}(t, k)$. With the help of the formula of total probability we obtain the equalities:

$$
\begin{aligned}
& \varphi_{n}(t, k)=\sum_{j=0}^{m-n} \int_{0}^{t} \mathbf{P}_{n}\{\eta(x)=j\} \varphi_{n+j-1}(t-x, k) d F(x)+ \\
& \quad+I\{n \leq k \leq m\} \mathbf{P}_{n}\{\eta(t)=k-n\} \bar{F}(t), \quad n \in\left\{1,2, \ldots, h_{2}\right\} ; \\
& \tilde{\psi}_{n}(t, k)=\sum_{j=0}^{m-n} \int_{0}^{t} \mathbf{P}_{n}\{\eta(x)=j\} \tilde{\psi}_{n+j-1}(t-x, k) d \tilde{F}(x)+ \\
& \quad+I\{n \leq k \leq m\} \mathbf{P}_{n}\{\eta(t)=k-n\} \overline{\tilde{F}}(t), \quad n \in\left\{h_{1}+1, h_{1}+2, \ldots, m\right\} .
\end{aligned}
$$


Here $I\{A\}$ is the indicator of a random event $A$; it equals 1 or 0 depending on whether or not the event $A$ occurs.

Introduce the notation:

$$
f_{(n)}(s, k, m)=I\{n \leq k \leq m\} q_{n, k-n}(s), \quad \tilde{f}_{(n)}(s, k, m)=I\{n \leq k \leq m\} \tilde{q}_{n, k-n}(s) .
$$

Taking into account the relations (1) and (2), from (7) we obtain the system of equations for the functions $\Phi_{n}(s, k)$ and $\tilde{\Phi}_{n}(s, k)$ :

$$
\begin{gathered}
\Phi_{n}(s, k)=f(s) \sum_{j=0}^{m-n} \pi_{n, j-1}(s) \Phi_{n+j-1}(s, k)+f_{(n)}(s, k, m), \quad n \in\left\{1,2, \ldots, h_{2}\right\}, \\
\tilde{\Phi}_{n}(s, k)=\tilde{f}(s) \sum_{j=0}^{m-n} \tilde{\pi}_{n, j-1}(s) \tilde{\Phi}_{n+j-1}(s, k)+\tilde{f}_{(n)}(s, k, m), \quad n \in\left\{h_{1}+1, h_{1}+2, \ldots, m\right\},
\end{gathered}
$$

with the boundary conditions

$$
\Phi_{0}(s, k)=0, \quad \tilde{\Phi}_{h_{1}}(s, k)=\Phi_{h_{1}}(s, k) .
$$

For solving the systems of equations (8)-(10) we will use the functions $R_{n i}(s)$ and $\tilde{R}_{n i}(s)$, defined by the recurrence relations:

$$
\begin{array}{r}
R_{n 1}(s)=R_{n+1}(s) ; \quad R_{n, j+1}(s)=R_{n+1}(s)\left(R_{n+1, j}(s)-f(s) \sum_{i=0}^{j-1} \pi_{n+1, i}(s) R_{n+1+i, j-i}(s)\right), \\
n \in\left\{0,1,2, \ldots, h_{2}-1\right\}, j \in\{1,2, \ldots, m-n-1\} ; \\
\tilde{R}_{n 1}(s)=\tilde{R}_{n+1}(s) ; \quad \tilde{R}_{n, j+1}(s)=\tilde{R}_{n+1}(s)\left(\tilde{R}_{n+1, j}(s)-\tilde{f}(s) \sum_{i=0}^{j-1} \tilde{\pi}_{n+1, i}(s) \tilde{R}_{n+1+i, j-i}(s)\right), \\
n \in\left\{h_{1}, h_{1}+1, h_{1}+2, \ldots, m-1\right\}, j \in\{1,2, \ldots, m-n-1\} .
\end{array}
$$

Introduce the notation:

$$
\begin{aligned}
& C_{n}(s)=R_{n, h_{2}-n}(s)-f(s) \sum_{i=1}^{h_{2}-n} R_{n i}(s) \pi_{n+i, h_{2}-n-i}(s), \quad C_{h_{2}}(s)=R_{h_{2}, 0}(s) \equiv 1 ; \\
& \tilde{D}_{n}(s)=-f(s) \sum_{i=1}^{h_{2}-n} R_{n i}(s) \sum_{j=h_{2}+1}^{m-1} \pi_{n+i, j-n-i}(s) \tilde{R}_{j, m-j}(s), \quad \tilde{D}_{h_{2}}(s) \equiv 0 ; \\
& D_{n}(s, k)=\sum_{i=1}^{h_{2}-n} R_{n i}(s) f_{(n+i)}(s, k, m)- \\
& -f(s) \sum_{i=1}^{h_{2}-n} R_{n i}(s) \sum_{j=h_{2}+1}^{m-1} \pi_{n+i, j-n-i}(s) \sum_{u=1}^{m-j} \tilde{R}_{j u}(s) \tilde{f}_{(j+u)}(s, k, m), \quad D_{h_{2}}(s, k) \equiv 0 .
\end{aligned}
$$


Theorem 1. For all $k \in\{1,2, \ldots, m\}$ and $\operatorname{Re} s>0$ the equalities

$$
\begin{array}{r}
\Phi_{n}(s, k)=\frac{1}{C_{0}(s)}\left(C_{n}(s) D_{0}(s, k)+\left(C_{0}(s) \tilde{D}_{n}(s)-C_{n}(s) \tilde{D}_{0}(s)\right) \Phi_{m}(s, k)\right)- \\
-D_{n}(s, k), \quad 1 \leq n \leq h_{2} ; \\
\Phi_{n}(s, k)=\tilde{R}_{n, m-n}(s) \Phi_{m}(s, k)-\sum_{i=1}^{m-n} \tilde{R}_{n i}(s) \tilde{f}_{(n+i)}(s, k, m), \quad h_{2}+1 \leq n \leq m-1 ; \\
\tilde{\Phi}_{n}(s, k)=\tilde{R}_{n, m-n}(s) \Phi_{m}(s, k)-\sum_{i=1}^{m-n} \tilde{R}_{n i}(s) \tilde{f}_{(n+i)}(s, k, m), \quad h_{1}+1 \leq n \leq h_{2}
\end{array}
$$

are fulfilled, where

$$
\Phi_{m}(s, k)=\frac{C_{h_{1}}(s) D_{0}(s, k)+C_{0}(s)\left(\sum_{i=1}^{m-h_{1}} \tilde{R}_{h_{1}}(s) \tilde{f}_{\left(h_{1}+i\right)}(s, k, m)-D_{h_{1}}(s, k)\right)}{C_{h_{1}}(s) \tilde{D}_{0}(s)+C_{0}(s)\left(\tilde{R}_{h_{1}, m-h_{1}}(s)-\tilde{D}_{h_{1}}(s)\right)} .
$$

Proof. Since $\Phi_{n}(s, k)=\tilde{\Phi}_{n}(s, k)$ for $n \in\left\{h_{2}+1, h_{2}+2, \ldots, m\right\}$, then the equalities (13) can be written as

$$
\tilde{\Phi}_{n}(s, k)=\tilde{R}_{n, m-n}(s) \tilde{\Phi}_{m}(s, k)-\sum_{i=1}^{m-n} \tilde{R}_{n i}(s) \tilde{f}_{(n+i)}(s, k, m), \quad h_{1}+1 \leq n \leq m-1 .
$$

Using the method of mathematical induction, equations (9), relations (11), and arguing as in the proof of Theorem 1 of [5], we obtain the proof of equalities (15).

The system of equations (8) can be written as

$$
\begin{aligned}
\Phi_{n}(s, k) & -f(s) \sum_{j=0}^{h_{2}-n} \pi_{n, j-1}(s) \Phi_{n+j-1}(s, k)=f(s) \sum_{j=h_{2}-n}^{m-n-1} \pi_{n j}(s) \Phi_{n+j}(s, k)+ \\
& +f_{(n)}(s, k, m), \quad 1 \leq n \leq h_{2} .
\end{aligned}
$$

We have received the equalities (15) from the equations (9). Similar arguments allow us to obtain from (16) the following relations:

$$
\begin{aligned}
& \Phi_{n}(s, k)=R_{n, h_{2}-n}(s) \Phi_{h_{2}}(s, k)- \\
& \quad-\sum_{i=1}^{h_{2}-n} R_{n i}(s)\left(f(s) \sum_{j=h_{2}}^{m-1} \pi_{n+i, j-n-i} \Phi_{j}(s, k)+f_{(n+i)}(s, k, m)\right), \quad 1 \leq n \leq h_{2}-1 .
\end{aligned}
$$

After defining with the help of $(15)$ all $\Phi_{n}(s, k)$ for $n \in\left\{h_{2}+1, h_{2}+2, \ldots, m-1\right\}$, the system (17) is rewritten as

$$
\Phi_{n}(s, k)=C_{n}(s) \Phi_{h_{2}}(s, k)+\tilde{D}_{n}(s) \Phi_{m}(s, k)-D_{n}(s, k), \quad 1 \leq n \leq h_{2}-1 .
$$


Using the boundary conditions (10), we can find $\Phi_{h_{2}}(s, k)$ and $\Phi_{m}(s, k)$. As a result, we obtain the equality

$$
\Phi_{h_{2}}(s, k)=\frac{1}{C_{0}(s)}\left(D_{0}(s, k)-\tilde{D}_{0}(s) \Phi_{m}(s, k)\right)
$$

and relations (12), (14). The theorem is proved.

\section{Busy period and stationary distribution}

If the system starts functioning at the moment when the first customer arrives, then

$$
\begin{aligned}
& \int_{0}^{\infty} e^{-s t} \mathbf{P}\{\xi(t)=k, \tau(m)>t\} d t=\Phi_{1}(s, k)= \\
& \quad=\frac{1}{C_{0}(s)}\left(C_{1}(s) D_{0}(s, k)+\left(C_{0}(s) \tilde{D}_{1}(s)-C_{1}(s) \tilde{D}_{0}(s)\right) \Phi_{m}(s, k)\right)-D_{1}(s, k) .
\end{aligned}
$$

To obtain a representation for $\int_{0}^{\infty} e^{-s t} \mathbf{P}\{\tau(m)>t\} d t$ we sum up equalities (18) for $k$ running from 1 to $m+1$. Given the definitions of $f_{(n)}(s, k, m), \tilde{f}_{(n)}(s, k, m)$, $q_{n j}(s)$ and $\tilde{q}_{n j}(s)$, it is not difficult to ascertain that

$$
\begin{aligned}
& \sum_{k=1}^{m} f_{(n)}(s, k, m)=\sum_{k=n}^{m} f_{(n)}(s, k, m)=\sum_{j=0}^{m-n} q_{n j}(s)=\frac{1-f(s)}{s}, \quad n \in\left\{1,2, \ldots, h_{2}\right\} ; \\
& \sum_{k=1}^{m} \tilde{f}_{(n)}(s, k, m)=\sum_{k=n}^{m} \tilde{f}_{(n)}(s, k, m)=\sum_{j=0}^{m-n} \tilde{q}_{n j}(s)=\frac{1-\tilde{f}(s)}{s}, \quad n \in\left\{h_{1}+1, h_{1}+2, \ldots, m\right\} .
\end{aligned}
$$

Introduce the notation:

$$
\begin{aligned}
& D_{n}(s)=\frac{1-f(s)}{s} \sum_{i=1}^{h_{2}-n} R_{n i}(s)-f(s) \frac{1-\tilde{f}(s)}{s} \sum_{i=1}^{h_{2}-n} R_{n i}(s) \sum_{j=h_{2}+1}^{m-1} \pi_{n+i, j-n-i}(s) \sum_{u=1}^{m-j} \tilde{R}_{j u}(s) ; \\
& \Phi_{m}(s)=\frac{C_{h_{1}}(s) D_{0}(s)+C_{0}(s)\left(\frac{1-\tilde{f}(s)}{s} \sum_{i=1}^{m-h_{1}} \tilde{R}_{h_{1}}(s)-D_{h_{1}}(s)\right)}{C_{h_{1}}(s) \tilde{D}_{0}(s)+C_{0}(s)\left(\tilde{R}_{h_{1}, m-h_{1}}(s)-\tilde{D}_{h_{1}}(s)\right)} .
\end{aligned}
$$

Thus, (18) confirms the following statement. 
Theorem 2. The Laplace transform of the distribution function of the length of the busy period is defined as

$$
\begin{aligned}
\int_{0}^{\infty} e^{-s t} \mathbf{P}\{\tau(m) & >t\} d t= \\
& =\frac{1}{C_{0}(s)}\left(C_{1}(s) D_{0}(s)+\left(C_{0}(s) \tilde{D}_{1}(s)-C_{1}(s) \tilde{D}_{0}(s)\right) \Phi_{m}(s)\right)-D_{1}(s) .
\end{aligned}
$$

To find $\int_{0}^{\infty} \mathbf{P}\{\tau(m)>t\} d t=\mathbf{E} \tau(m)$ we need to pass to the limit in (19) as $s \rightarrow+0$. We use the sequences $\pi_{n i}, R_{n}, \tilde{\pi}_{n i}$ and $\tilde{R}_{n}$, as well as sequences $R_{n i}$ and $\tilde{R}_{n i}$, obtained by limit passages: $R_{n i}=\lim _{s \rightarrow+0} R_{n i}(s), \tilde{R}_{n i}=\lim _{s \rightarrow+0} \tilde{R}_{n i}(s)$. For $R_{n i}$ and $\tilde{R}_{n i}(11)$ implies the recurrence relations:

$$
\begin{aligned}
& R_{n 1}=R_{n+1} ; \quad R_{n, j+1}= R_{n+1}\left(R_{n+1, j}-\sum_{i=0}^{j-1} \pi_{n+1, i} R_{n+1+i, j-i}\right), \\
& n \in\left\{0,1,2, \ldots, h_{2}-1\right\}, j \in\{1,2, \ldots, m-n-1\} ; \\
& \tilde{R}_{n 1}=\tilde{R}_{n+1} ; \quad \tilde{R}_{n, j+1}=\tilde{R}_{n+1}\left(\tilde{R}_{n+1, j}-\sum_{i=0}^{j-1} \tilde{\pi}_{n+1, i} \tilde{R}_{n+1+i, j-i}\right), \\
& n \in\left\{h_{1}, h_{1}+1, h_{1}+2, \ldots, m-1\right\}, \quad j \in\{1,2, \ldots, m-n-1\} .
\end{aligned}
$$

Using the relations (20) and taking into account the equalities

$$
\sum_{j=-1}^{m-n-1} \tilde{\pi}_{n j}=1, \quad n \in\left\{h_{1}+1, h_{1}+2, \ldots, m\right\}
$$

by mathematical induction we can prove that

$$
\tilde{R}_{n, m-n}=1, \quad n \in\left\{h_{1}+1, h_{1}+2, \ldots, m-1\right\} .
$$

Given (6) and (21), using (19) we obtain the following statement.

Theorem 3. The mean length of the busy period is determined in the form

$$
\mathbf{E} \tau(m)=M T\left(m, h_{1}, h_{2}\right)+\tilde{M} \tilde{T}\left(m, h_{1}, h_{2}\right),
$$

where 


$$
\begin{aligned}
& T\left(m, h_{1}, h_{2}\right)=\sum_{i=1}^{h_{2}} R_{0 i}-\sum_{i=1}^{h_{2}-1} R_{1 i}-R\left(h_{1}, h_{2}\right) \sum_{i=1}^{h_{2}-h_{1}} R_{h_{1}} ; \\
& \tilde{T}\left(m, h_{1}, h_{2}\right)=\left(\sum_{i=1}^{h_{2}-1} R_{1 i} \sum_{j=h_{2}+1}^{m-1} \pi_{i+1, j-i-1}-\sum_{i=1}^{h_{2}} R_{0 i} \sum_{j=h_{2}+1}^{m-1} \pi_{i, j-i}\right) \sum_{u=1}^{m-j} \tilde{R}_{j u}+ \\
& +R\left(h_{1}, h_{2}\right)\left(\sum_{i=1}^{h_{2}-h_{1}} R_{h_{1} i} \sum_{j=h_{2}+1}^{m-1} \pi_{h_{1}+i, j-h_{1}-i} \sum_{u=1}^{m-j} \tilde{R}_{j u}+\sum_{i=1}^{m-h_{1}} \tilde{R}_{h_{1} i}\right) ; \quad R\left(h_{1}, h_{2}\right)=\frac{R_{0, h_{2}+1}-R_{1 h_{2}}}{R_{h_{1}, h_{2}-h_{1}+1}}
\end{aligned}
$$

Introduce the notation: $\lim _{t \rightarrow \infty} \mathbf{P}\{\xi(t)=k\}=p_{k}(m), k \in\{0,1,2, \ldots, m\}$. Reasoning as in the paper [6], and taking into account that the length of the idle period during one cycle is distributed exponentially with parameter $m \lambda$, from (18) we obtain formulas for the stationary distribution of the number of customers in the system.

Theorem 4. The stationary distribution of the number of customers in the system is given by

$$
\begin{aligned}
& p_{0}(m)=\frac{1}{1+m \lambda \mathbf{E} \tau(m)} ; \\
& p_{k}(m)=m \lambda p_{0}(m)\left(R_{0 k} q_{k 0}+\sum_{i=1}^{k-1}\left(R_{0 i} q_{i, k-i}-R_{1 i} q_{i+1, k-i-1}\right)\right), \quad k \in\left\{1,2, \ldots, h_{1}\right\} ; \\
& p_{k}(m)=m \lambda p_{0}(m)\left(\sum_{i=1}^{k} R_{0 i} q_{i, k-i}+R\left(h_{1}, h_{2}\right) \sum_{i=1}^{k-h_{1}}\left(\tilde{R}_{h_{1}} \tilde{q}_{h_{1}+i, k-h_{1}-i}-R_{h_{1} l} q_{h_{1}+i, k-h_{1}-i}\right)-\right. \\
&\left.-\sum_{i=1}^{k-1} R_{1 i} q_{i+1, k-1-i}\right), \quad k \in\left\{h_{1}+1, h_{1}+2, \ldots, h_{2}\right\} ; \\
& p_{k}(m)=m \lambda p_{0}(m)\left(D_{0}(k)+R\left(h_{1}, h_{2}\right)\left(\sum_{i=1}^{k-h_{1}} \tilde{R}_{h_{1}} \tilde{q}_{h_{1}+i, k-h_{1}-i}-D_{h_{1}}(k)\right)-D_{1}(k)\right), \\
& k \in\left\{h_{2}+1, h_{2}+2, \ldots, m\right\},
\end{aligned}
$$

where

$$
D_{n}(k)=\sum_{i=1}^{h_{2}-n} R_{n i}\left(q_{n+i, k-n-i}-I\left\{h_{2}+2 \leq k \leq m\right\} \sum_{j=h_{2}+1}^{k-1} \pi_{n+i, j-n-i} \sum_{u=1}^{k-j} \tilde{R}_{j u} \tilde{q}_{j+u, k-j-u}\right) .
$$

We find the stationary queue characteristics - the average queue length $\mathbf{E} Q(m)$ and average waiting time $\mathbf{E} w(m)$ - by the formulas 


$$
\mathbf{E} Q(m)=\sum_{k=1}^{m-1} k p_{k+1}(m) ; \quad \mathbf{E} w(m)=\frac{\mathbf{E} Q(m)}{\lambda_{\mathrm{av}}} .
$$

Here $\lambda_{\mathrm{av}}$ is a steady-state value of the arrival rate of customers, defined by the equality

$$
\lambda_{\mathrm{av}}=\sum_{k=0}^{m-1}(m-k) p_{k}(m)
$$

The parameter $\lambda_{\mathrm{av}}$ is characteristic of the system capacity, because for the steady-state regime we have the equality of the intensities of flows of customers arriving and served.

If $\tilde{F}(x)=F(x)$, then we obtain a closed system with the distribution of the service time that is independent of the number of customers in the system. Putting $h_{1}=h_{2}=m$ in (22) and (23), we obtain formulas for $\mathbf{E} \tau(m)$ and $p_{k}(m)$ for this system.

\section{Examples for calculating of stationary characteristics}

Let us consider two examples for the calculation of stationary characteristics of closed queueing systems.

Example 1. Assume that $m=6, \lambda=2, h_{1}=2, h_{2}=4$, the uniform distribution on the intervals $(0 ; 0.5]$ and $(0 ; 0.25]$ corresponds to the distribution functions of the service time $F(x)$ and $\tilde{F}(x)$ respectively. Thus,

$$
M=0.25, \quad \tilde{M}=0.125, \quad f(y)=\frac{2}{y}\left(1-e^{-0.5 y}\right), \quad \tilde{f}(y)=\frac{4}{y}\left(1-e^{-0.25 y}\right) .
$$

Example 2. Consider a closed system for which $\tilde{F}(x)=F(x)$. Assume that the distribution function $F(x)$ and the values of parameters $m, \lambda, h_{1}$ and $h_{2}$ are the same as in Example 1.

Let us call system $i$ a system with parameters corresponding to the example $i$, $i=1,2$.

The row " $p_{k}(6) "$ of Tables 1 and 2 contains steady-state probabilities $p_{k}(6)$, calculated by the formulas (23) for the systems 1 and 2 respectively. For the sake of comparison, the same tables contain the corresponding probabilities evaluated by the GPSS World simulation system [13] for the time value $t=10^{6}$. The values of the stationary characteristics found by the formulas (22), (24) and (25) and calculated with the help of GPSS World, are shown in Tables 3 and 4 respectively. 
Stationary distributions of the number of customers in the system 1

\begin{tabular}{|l|c|c|c|c|c|c|c|}
\hline $\begin{array}{c}\text { Number } \\
\text { of customers }(k)\end{array}$ & 0 & 1 & 2 & 3 & 4 & 5 & 6 \\
\hline$p_{k}(6)$ & 0.00709 & 0.03431 & 0.12376 & 0.26156 & 0.33006 & 0.19865 & 0.04455 \\
\hline $\begin{array}{c}p_{k}(6) \\
\left.\text { (GPSS World, } t=10^{6}\right)\end{array}$ & 0.00716 & 0.03444 & 0.12346 & 0.26220 & 0.32932 & 0.19931 & 0.04412 \\
\hline
\end{tabular}

Table 2

Stationary distributions of the number of customers in the system 2

\begin{tabular}{|l|c|c|c|c|c|c|c|}
\hline $\begin{array}{c}\text { Number } \\
\text { of customers }(k)\end{array}$ & 0 & 1 & 2 & 3 & 4 & 5 & 6 \\
\hline$p_{k}(6)$ & 0.00450 & 0.02176 & 0.07848 & 0.20107 & 0.32825 & 0.28159 & 0.08435 \\
\hline $\begin{array}{l}p_{k}(6) \\
\left(\text { GPSS World, } t=10^{6}\right)\end{array}$ & 0.00457 & 0.02183 & 0.07839 & 0.20129 & 0.32779 & 0.28188 & 0.08425 \\
\hline
\end{tabular}

Table 3

Stationary characteristics of the system 1

\begin{tabular}{|l|c|c|c|c|}
\hline \multicolumn{1}{|c|}{ Characteristics } & $\mathbf{E} \tau(6)$ & $\mathbf{E} Q(6)$ & $\mathbf{E} w(6)$ & $\lambda_{\text {av }}$ \\
\hline Analytical value & 11.673 & 2.654 & 0.564 & 4.705 \\
\hline Value according to GPSS World, $t=10^{6}$ & 11.613 & 2.653 & 0.564 & 4.707 \\
\hline
\end{tabular}

Table 4

Stationary characteristics of the system 2

\begin{tabular}{|l|c|c|c|c|}
\hline \multicolumn{1}{|c|}{ Characteristics } & $\mathbf{E} \tau(6)$ & $\mathbf{E} Q(6)$ & $\mathbf{E} w(6)$ & $\lambda_{\text {av }}$ \\
\hline Analytical value & 18.456 & 3.013 & 0.757 & 3.982 \\
\hline Value according to GPSS World, $t=10^{6}$ & 18.264 & 3.013 & 0.756 & 3.982 \\
\hline
\end{tabular}

Analyzing the obtained results, we see that the application of the hysteretic strategy of the service time change allows one to reduce the queue length and the length of the busy period and, therefore, to increase the capacity of the system $\lambda_{\mathrm{av}}$.

\section{Conclusions}

With the help of the potentials method, we have obtained simple and suitable for numerical realization formulas for finding the stationary characteristics of single-channel closed systems with an arbitrary distribution of the service time. 
In order to increase the system capacity, the hysteretic strategy of the service time change are applied.

\section{References}

[1] Nesterov Yu.G., Analysis of characteristics of a closed queuing system with relative priorities, Nauka i Obrazovanie, MGTU im. N. Baumana 2014, 3, 242-254 (in Russian).

[2] Bocharov P.P., Pechinkin A.V., Queueing Theory, RUDN, Moscow 1995 (in Russian).

[3] Zhernovyi Yu.V., Markov Queueing Models, Vydavnychyi Tsentr LNU im. Ivana Franka, Lviv 2004 (in Ukrainian).

[4] Zhernovyi Yu., Stationary probability distribution of states for a single-channel closed queuing system, Visnyk Lviv. Univer. Series Mech.-Math. 2007, 67, 130-136 (in Ukrainian).

[5] Zhernovyi Yu.V., Zhernovyi K. Yu., Potentials method for a closed system with service times dependent on the queue length, Informatsionnye Protsessy 2015, 15, 1, 40-50.

[6] Zhernovyi K.Yu., Zhernovyi Yu.V., $\mathrm{M}^{\theta} / \mathrm{G} / 1 / \mathrm{m}$ and $\mathrm{M}^{\theta} / \mathrm{G} / 1$ systems with the service time dependent on the queue length, J. of Communicat. Technology and Electronics 2013, 58, 12, 1267-1275.

[7] Zhernovyi Yu., Zhernovyi K., Potentials Method for Threshold Strategies of Queueing, LAP Lambert Academic Publishing, Saarbrücken 2015 (in Russian).

[8] Zhernovyi K.Yu., Stationary characteristics of the $\mathrm{M}^{\theta} / \mathrm{G} / 1 / \mathrm{m}$ system with the threshold functioning strategy, J. of Communicat. Technology and Electronics 2011, 56, 12, 1585-1596.

[9] Zhernovyi Yu., Insensitivity of the Queueing Systems Characteristics, LAP Lambert Academic Publishing, Saarbrücken 2015.

[10] Zhernovyi Yu.V., Zhernovyi K.Yu., Probabilistic characteristics of an $\mathrm{M}_{2}^{\theta} / \mathrm{G} / 1 / \mathrm{m}$ queue with two-loop hysteretic control of the service time and arrival rate, J. of Communicat. Technology and Electronics 2014, 59, 12, 1465-1474.

[11] Korolyuk V.S., The Boundary Problem for the Compound Poisson Processes, Naukova Dumka, Kyiv 1975.

[12] Bratiychuk M., Borowska B., Explicit formulae and convergence rate for the system $M^{\alpha} / G / 1 / N$ as $N \rightarrow \infty$, Stochastic Models 2002, 18, 1, 71-84.

[13] Zhernovyi Yu., Creating Models of Queueing Systems in the Environment GPSS World, Palmarium Academic Publishing, Saarbrücken 2014 (in Russian). 